\title{
CHALLENGES IN NANOMETROLOGY: HIGH PRECISION MEASUREMENT OF POSITION AND SIZE
}

\author{
Harald Bosse, Bernd Bodermann, Gaoliang Dai, Jens Flügge, Carl Georg Frase, \\ Wolfgang Häßler-Grohne, Paul Köchert, Rainer Köning, Christoph Weichert
}

Physikalisch-Technische Bundesanstalt (PTB), Bundesallee 100, 38116 Braunschweig, Germany

\begin{abstract}
This contribution describes recent developments of the PTB in high precision position and size metrology as support for different nanotechnology applications. It will be shown how measurement uncertainties of about 1-2 nm for 1D-position of graduation lines on $152 \mathrm{~mm}$ photomasks (6"), or on line scales and incremental encoders of about $300 \mathrm{~mm}$ length have been achieved. The measurement of the size of nanoscale features represents additional challenges, because in addition to the length metric, also the location of opposite feature edges has to be precisely determined. In addition to other feature size or CD metrology techniques applied at PTB, a recent development which uses transmission electron microscopy in the traceability chain of AFM CD measurements will be described. The uncertainties achieved for CD measurements on high quality Si line structures are $U_{95 \%}=$ $1.6 \mathrm{~nm}$.
\end{abstract}

Index Terms - traceability, measurement uncertainty, New SI, interferometry, line scale length encoder, straightness, photomask, CD metrology, signal modeling, reference

\section{INTRODUCTION}

The precise measurement of the position of an object to be measured or machined is a basic task in precision engineering applications. If features of nanoscale dimensions are of interest this task is within the area of nanometrology. In a broader sense this also holds for features with larger dimensions if the machining tolerances or the required measurement uncertainties are in the nm range.

This contribution describes recent developments of the PTB in high precision position and size metrology as support for different nanotechnology applications. The most stringent requirements on the measurement uncertainty for position and size of nanoscale feature are defined by lithography technologies, as described in the regularly updated International Technology Roadmap for Semiconductors (ITRS).

For high precision measurement of the position of lithographic features, the PTB has developed the so-called Nanometer Comparator, a 1D displacement comparator based on vacuum interferometry. This comparator has been further improved over the years and these developments as well as examples of the latest measurement performance will be presented. For example, optical microscopic measurements of the positions of line graduations on 152 mm (6") photomasks can be made with an uncertainty of $U_{95 \%}=2 \mathrm{~nm}$ over $140 \mathrm{~mm}$ and measurements on high quality 1D incremental length encoders of $500 \mathrm{~mm}$ length $l$ can be performed with an uncertainty of $U_{95 \%}=\left[(1 \mathrm{~nm})^{2}+\left(2 \cdot 10^{-9} \cdot l\right)^{2}\right]^{1 / 2}$.

The Nanometer comparator has been upgraded by a new object carriage and a special straightness interferometer to also allow straightness measurements to be performed e.g. on 
photomasks carrying cross mark graduations or length encoders carrying additional straightness graduations (1.5D encoders). Details of these developments as well as planned improvements in the drive system of the Nanometer Comparator will be described.

In another application a specially designed highly symmetrical heterodyne optical interferometer is used in a combined optical and $\mathrm{x}$-ray interferometer, made of ${ }^{28} \mathrm{Si}$ material in order to calibrate the ${ }^{28} \mathrm{Si}$ lattice plane spacing with an uncertainty below $2 \cdot 10^{-9}$. This experiment is part of a set of different high precision experiments, which should allow to determine a numerical value for the Avogadro constant $N_{\mathrm{A}}$ with an uncertainty below $2 \cdot 10^{-8}$. The precise determination of the Avogadro constant $N_{\mathrm{A}}$ in addition to three other natural constants, namely Planck constant $h$, Boltzmann constant $k$ and elementary charge $e$ is important for the envisaged redefinition of the International System of Units, the SI.

The measurement of the size of nanoscale features presents additional challenges, because in addition to the length metric, also the location of opposite feature edges has to be precisely determined. As in other cases of precision engineering, these bidirectional measurands, like e.g. the width of a line (critical dimension, CD) or the diameter of a circle or aperture need suitable physical modeling of the sensor signal (optical microscope, scanning electron microscope or AFM), which is used for location of the feature edges. In addition to other feature size or CD metrology techniques applied at PTB, a recent development which uses transmission electron microscopy in the traceability chain of AFM CD measurements will be described. The uncertainties achieved for $\mathrm{CD}$ measurements on high quality Si line structures are $U_{95 \%}=1.6 \mathrm{~nm}$.

\section{HIGH PRECISION MEASUREMENT OF FEATURE POSITION}

High precision manufacturing requires special positioning capabilities in order to guarantee the applied structuring process takes place at the intended position on the workpiece to be machined or the substrate to be patterned. The required positioning accuracy can normally only be realized by interferometric position measurement systems, such as laser displacement interferometers or grating-based length encoder systems. For calibration of such length measurement systems and of graduated length measurement standards as well, such as line scales or photomasks, the PTB has developed the so-called Nanometer Comparator, a 1D reference length comparator using vacuum interferometry, which will be explained in greater detail in section 2.1 .

For determination of the lattice parameter of ${ }^{28} \mathrm{Si}$ single crystal material with a target uncertainty of $2 \cdot 10^{-9}$ a special vacuum displacement interferometer has been developed, which will be combined with an X-ray interferometer made of the ${ }^{28} \mathrm{Si}$ material. A detailed description of this challenging task in high precision displacement measurement will be provided in section 2.2 .

\subsection{Nanometer Comparator}

The so-called Nanometer Comparator is the national standard for high precision 1D length calibrations of length standards and measuring instruments with graduations such as line scales, photomasks or length encoder systems. The comparator uses a heterodyne interferometer in vacuum. The device under test, however, is kept under ambient conditions to avoid the correction of the length changes introduced by the ambient pressure, which is about $0.5 \mu \mathrm{m} / \mathrm{bar}$ and would require the sample's compressibility to be well known. The sample is located on a moving carriage guided by air bearings in $y$ - and z-direction and driven in $\mathrm{x}$ direction by a linear motor. The comparator was jointly developed with Heidenhain company and was installed at the PTB in the year 2000. Figure 1 shows a scheme of the Nanometer 
Comparator, while fig. 2 illustrates the measurement loop of the Nanometer Comparator using four different interferometer beams and the compensation principle of the residual guiding deviations, for further details see [1].

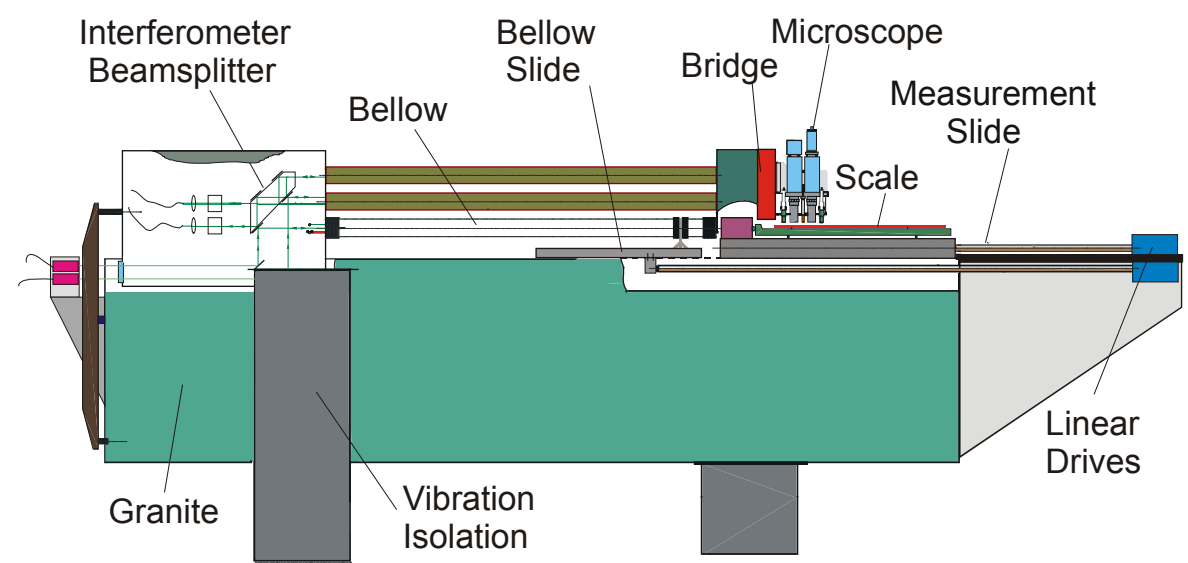

Fig. 1: Basic design of the Nanometer Comparator.

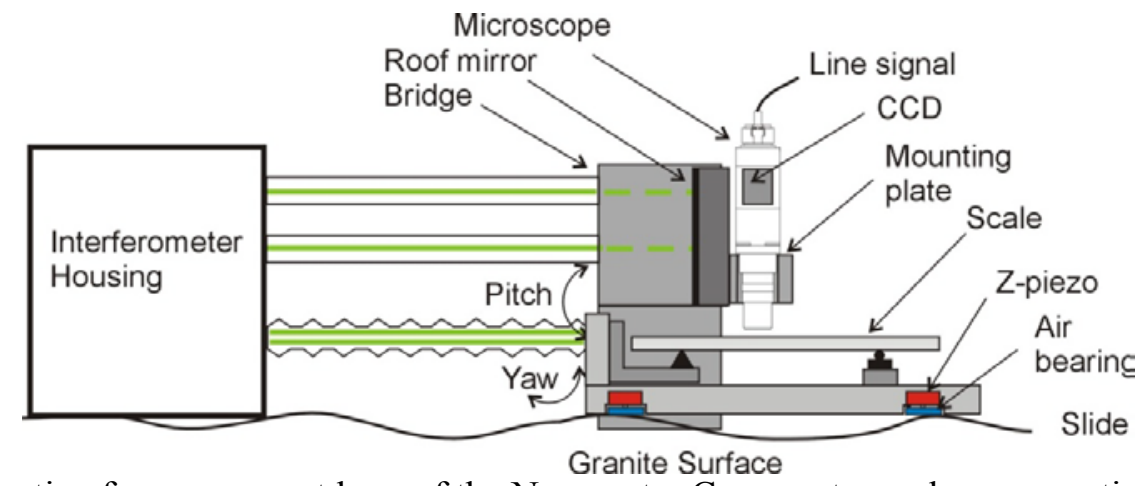

Fig. 2: Schematic of measurement loop of the Nanometer Comparator and compensation principle of residual guiding deviations. The single green line in the vacuum bellow represents two beams.

The performance of the Nanometer Comparator was proven in an international comparison of three vacuum length comparators using a $280 \mathrm{~mm}$ incremental length encoder as a transfer standard [2]. The measurement results of this comparison agreed within $2 \cdot 10^{-8}$. The measurement uncertainty of the Nanometer Comparator on length encoder systems of measurement length $l$ has been determined to: $U_{95 \%}=\left[(1 \mathrm{~nm})^{2}+\left(2 \cdot 10^{-9} \cdot l\right)^{2}\right]^{1 / 2}$. But it requires a high quality grating produced on a low thermal expansion substrate of sufficient thickness and good flatness deviation to be used by the encoder system.
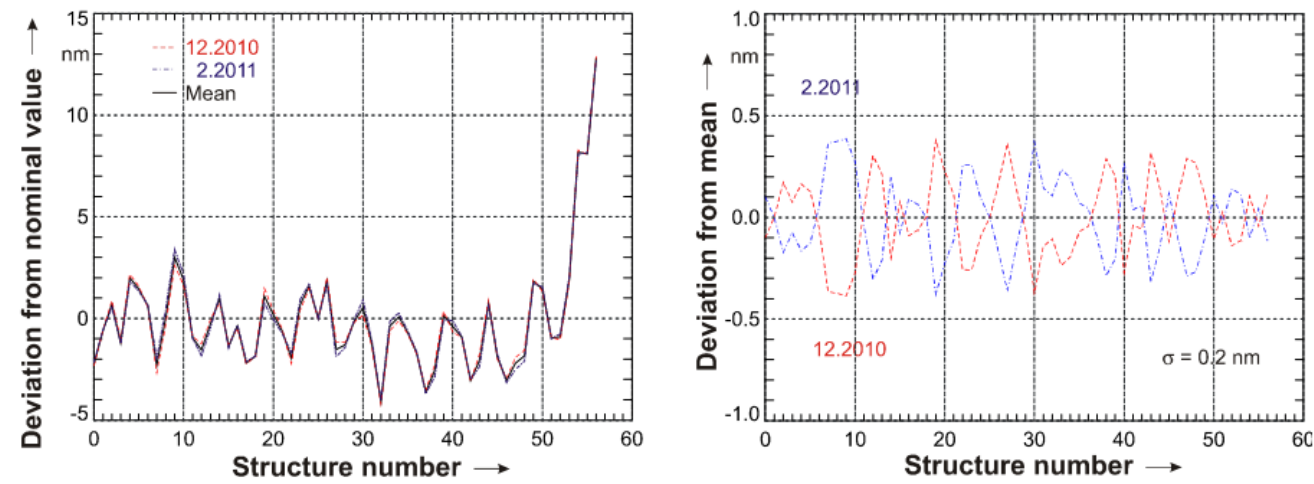

Fig. 3: Measurement results for the position deviations of graduation lines on a photomask (6") over a total measurement length of $140 \mathrm{~mm}$ : left: deviations from nominal values, right: deviations from common mean. 
The reproducibility level of the Nanometer Comparator for position measurements on photomasks with high quality line graduations is illustrated in fig. 3. It remains below $0.5 \mathrm{~nm}$ and exhibits a $1 \sigma$ standard deviation of $0.2 \mathrm{~nm}$ [3]. The measurement uncertainty for line positions on high quality 6" photomasks (chrome on quartz or MoSi) currently is $2 \mathrm{~nm}$.

Within a recent upgrade a new measurement object carrier has been integrated in the Nanometer Comparator setup. It is made from Zerodur and also provides a long plane mirror along the $\mathrm{x}$-measurement axis of the comparator. It allows to add a special straightness interferometer, which measures in the y-direction and which uses the plane mirror for detection of straightness deviations of the x-guideway. It is shown in fig. 4 . With the implementation of this additional measurement functionality, we have extended the capabilities of the Nanometer Comparator from a pure 1D displacement comparator to a 1.5D comparator for displacement and additional reference-free straightness metrology using the so-called TMS (traceable multiple sensor) method [4]. The straightness metrology capabilities are of use for calibration of the straightness deviations of patterns on photomasks or linear scales with cross marks and for so-called 1.5D encoder measurement systems which also provide straightness information in addition to the main displacement measurement axis.
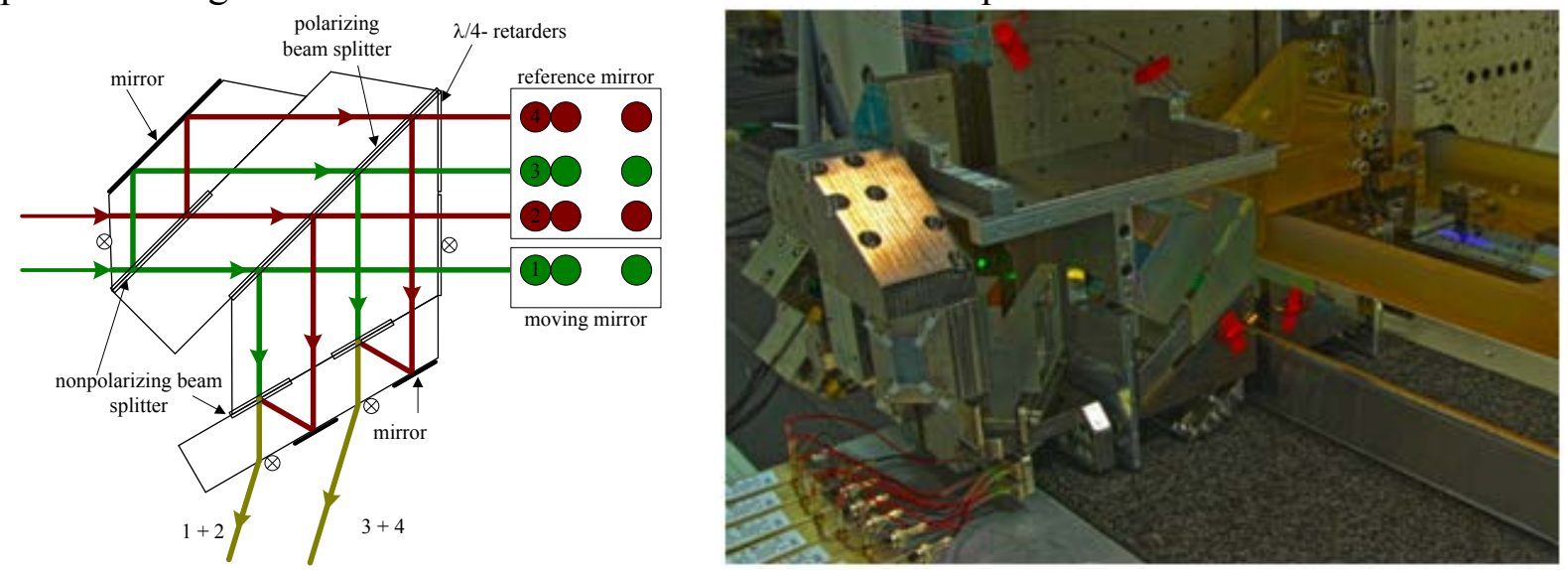

Fig. 4: Scheme of the straightness interferometer used for reference-free straightness metrology (left) and a photo of the Nanometer Comparator with integrated new Zerodur object holder and straightness interferometer (right).

First investigations of a special encoder system with a straightness measurement graduation have proven the high resolution, stability and small periodic non-linearity of the newly developed y-interferometers. Figure 5 shows the difference of the measurement results of the straightness encoder system and the y-interferometers measuring under ambient conditions over $200 \mathrm{~s}$. Using a sampling rate of $50 \mathrm{kHz}$, a standard deviation of $0.3 \mathrm{~nm}$ could be achieved. The residual periodic non-linearity of the y-interferometer, mainly caused by multireflections on the interfaces of the optical components could be proven to be below $0.3 \mathrm{~nm}$ in independent investigations [5].

Another direction of further optimization of the Nanometer Comparator relates to the coupling of the carriage to the linear motor drive which is guided by an independent air bearing. To adapt to slightly different orientations of the two linear guides, a rotationally symmetric flexure hinge has been used so far. This connection, however, also limits the stiffness between the drive system and the carriage in the x-direction. Effects of this limited stiffness are the observed vibrations of the carriage in x-direction with amplitudes of up to $15 \mathrm{~nm}$ and a resulting limited bandwidth of the carriage position control of about $25-30 \mathrm{~Hz}$. 


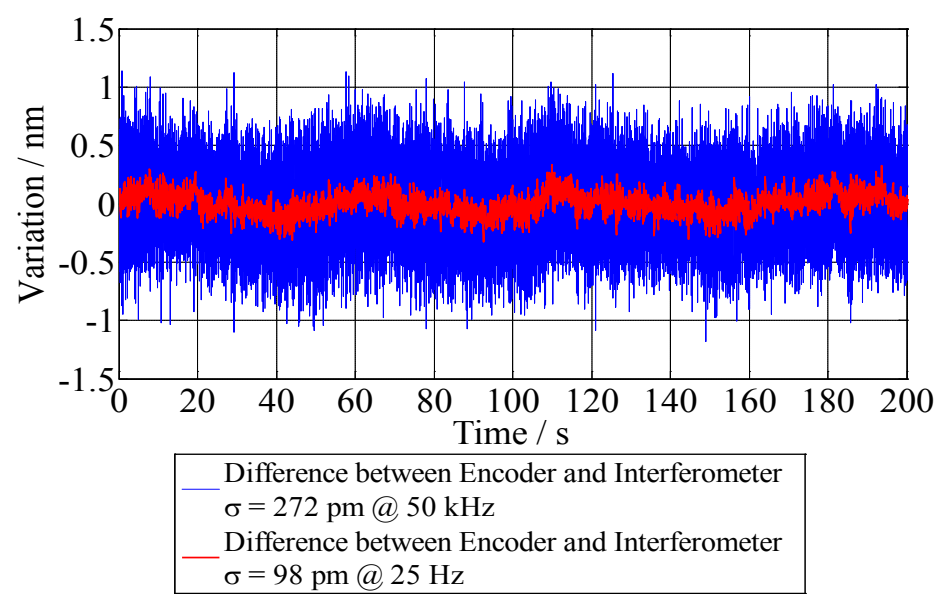

Fig. 5: Difference between results of y-interferometer and straightness encoder.

These mechanical vibrations of the measurement carriage also occur with damped amplitudes in the differential measurement results between a length encoder as measurement object and the vacuum displacement interferometer, although both systems were sampled highly synchronously. This is the reason, why we started to replace the rather weak mechanical coupling by a Lorentz actuator (voice coil actuator), which is directly controlled by the vacuum displacement interferometer signals.

In a test set-up shown in Figure 6(a), the realization of position stabilities in the sub-nm range using a FPGA-based control could be demonstrated. Here we only used components, which were also intended for the later integration into the Nanometer Comparator: the same Lorentz actuator, a high-dynamic position control and highly precise laser interferometric position feedback signals [6]. Figure 6(b) shows the obtained position stability of the test set-up.
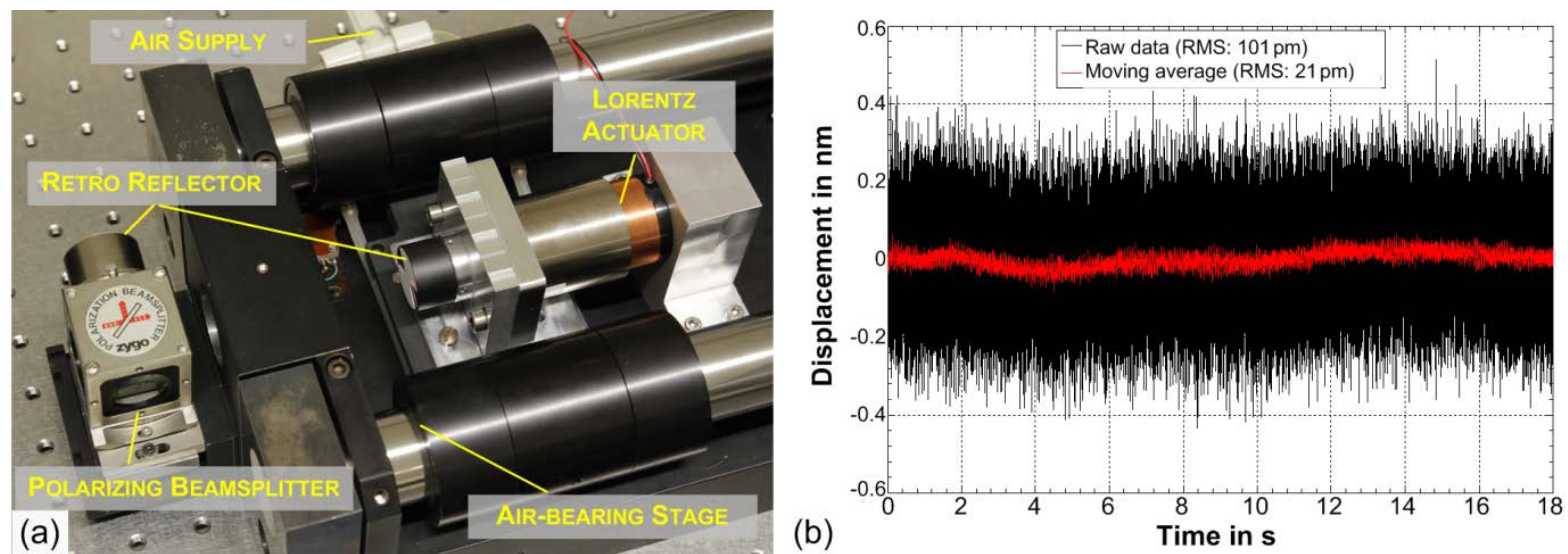

Fig. 6: (a) Experimental test-setup to prove the position stability of an air-bearing stage by tracking the position by a laser-interferometer. (b) Measurement results for position stability of the test set-up (over $18 \mathrm{~s}$ with control rate of $200 \mathrm{kHz}$, [6]): position stability of $0.1 \mathrm{~nm}$ (standard deviation of black curve) and a peak-to valley value of $1 \mathrm{~nm}$. The red curve shows a stability of $21 \mathrm{pm}$ for the moving average over 555 measurement values (bandwidth: $100 \mathrm{~Hz}$ ).

\subsection{High precision displacement measurement for a basic metrology task}

Several challenging manufacturing and metrology tasks have to be solved, before the proposed re-definition of the International System of Units, the SI may take place [7]. One of the challenges is related to the precise determination of the Avogadro constant [8]. Within this task the lattice plane spacing of isotope enriched ${ }^{28} \mathrm{Si}$ material needs to be determined experimentally using a combination of an x-ray interferometer with a high precision optical interferometer [9] with a relative uncertainty of about $2 \cdot 10^{-9}$. For a displacement of a 
separated X-ray interferometer (two ${ }^{28} \mathrm{Si}$ crystals) of about $5 \mathrm{~mm}$, this requirement transfers to a target uncertainty of the measurement of the moving part of the x-ray interferometer with an optical interferometer to be below $10 \mathrm{pm}$. It was already proven that a newly developed optical interferometer of the PTB achieved residual periodic non-linearity deviations, which are considered to provide the dominating uncertainty contribution below $10 \mathrm{pm} \mathrm{[10],} \mathrm{see}$ fig. 7.
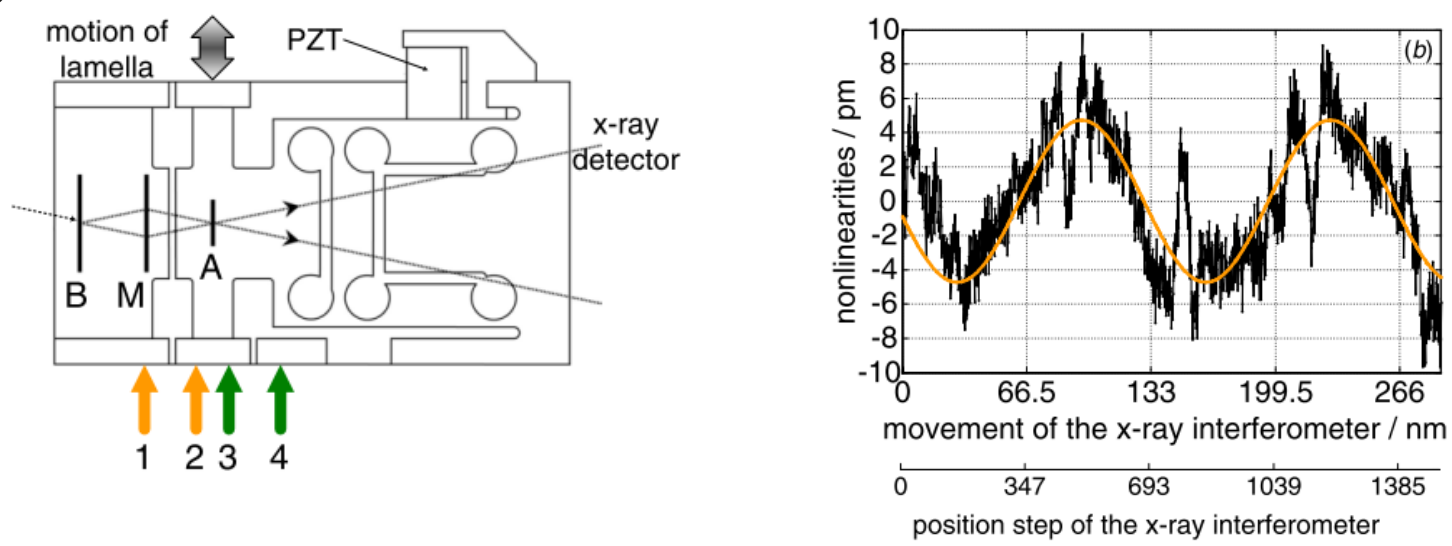

Fig. 7: Principle scheme of the combined $\mathrm{x}$-ray and optical interferometer for determination of the Si lattice plane spacing (left) and measurement result for the residual periodic non-linearity of the optical interferometer signal in comparison to the displacement measured by the $\mathrm{x}$-ray interferometer.

At PTB, currently a new setup for the combined x-ray and optical interferometer under vacuum conditions targeting at the above mentioned stringent overall uncertainty requirements for calibration of the ${ }^{28} \mathrm{Si}$ lattice plane spacing is being implemented.

\section{HIGH PRECISION MEASUREMENT OF FEATURE SIZE}

The measurements of the size of features, such as the width or critical dimension (CD) of a lithographic line feature or the diameter of a thin chromium circle on a (transparent) substrate are typical measurement tasks in nanometrology. Here it is important to exactly define the measurand, which could be e.g. the size at the top edge of a feature (see fig. 8) or the size at $50 \%$ height of the feature or at the bottom of the feature. These measurands normally result in different measurement results, because the sidewall characteristics of the feature edges usually are more complex than for an ideal vertical edge with negligible edge corner radius at the top and bottom of the sidewall.

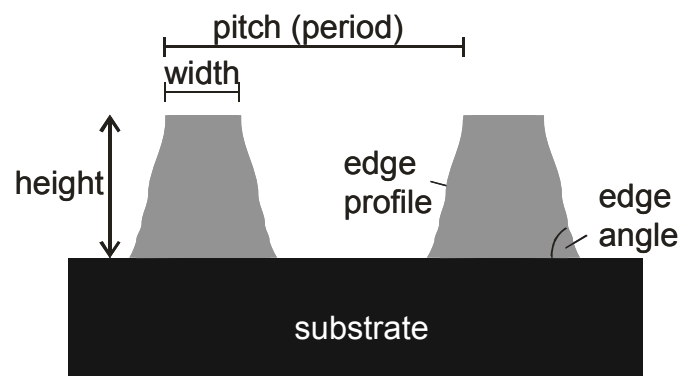

Fig. 8: Schematic representation of important dimensional measurands of micro- and nanostructures.

For all of the different measurement methods which are used for characterization of nanostructures the influence of the sidewall characteristics, with respect to topography as well as material, on the measurement signal have to be properly taken into account. The determination of the abovementioned measurands for the size of nanostructures can only be 
realized with small uncertainties if suitable physical models for analysis of the measurement signal at the feature edges are applied.

\subsection{Optical microscopy}

The imaging properties in optical microscopy including the resolution limitation by diffraction at the object features were first described by E. Abbe in 1873 [11]. For the calculation of the diffracted electromagnetic fields, an appropriate geometric model of the structure has to be assumed. If an electromagnetic plane wave irradiates the structured object, a part of the light is reflected or transmitted into the diffracted orders. The rigorous diffraction spectrum can be calculated numerically using different theoretical models. For the numeric solution of the stationary Maxwell equation, both the rigorous coupled wave analysis (RCWA) and the finite element method (FEM) are used at PTB. The RCWA method is based on a Fourier expansion of the permittivity function and of the electromagnetic fields. It was initially developed only for binary gratings. Arbitrary geometric profiles can be approximated by a staircase profile, but for each staircase an eigenvalue problem has to be solved.

The FEM method has the advantage that arbitrary geometries can be adapted and it shows fast convergence. The RCWA method calculates diffraction spectra of periodically continued structures only. Single structures are approximated by increasing the period so that the images of adjacent structures do not overlap. Typically the period is chosen in this case to be $\geq 10 \cdot \lambda$ where $\lambda$ is the optical wavelength. The RCWA simulations are computed with the software package MicroSim, which has been developed at the Institut für Technische Optik, Stuttgart, Germany [12]. For the FEM method, either the software package DIPOG from the Weierstrass Institute (WIAS) in Berlin [13], or JCM-Suite developed at the Zuse Institute Berlin (ZIB) is used [14].

At PTB, different microscope systems are used for feature width metrology. Figure 9 shows a UV transmission microscopy system, which has been described in detail in [15]. The achievable measurement uncertainty of this UV microscope system for CD on lithographic features of high quality on photomasks has been determined to be $U_{95 \%}=19 \mathrm{~nm}$. A DUV transmission microscope using $193 \mathrm{~nm}$ illumination wavelength targeting at uncertainty values of $U_{95 \%}=10 \mathrm{~nm}$ and below has been described in [16]. An overview of optical microscopy at the PTB including comparison measurements for validation of the applied imaging models has been published in [17].

a)

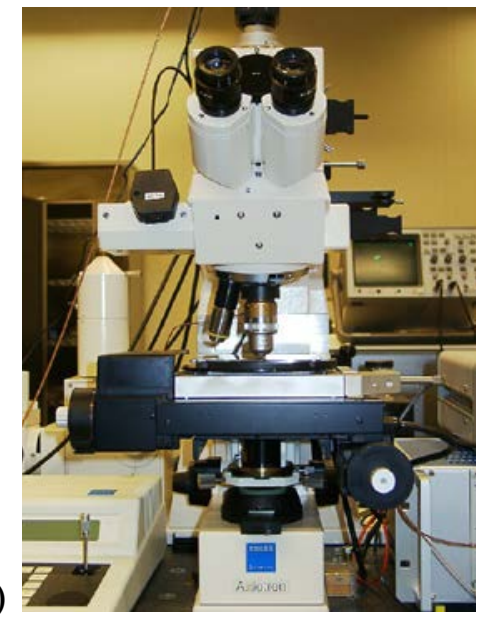

b)

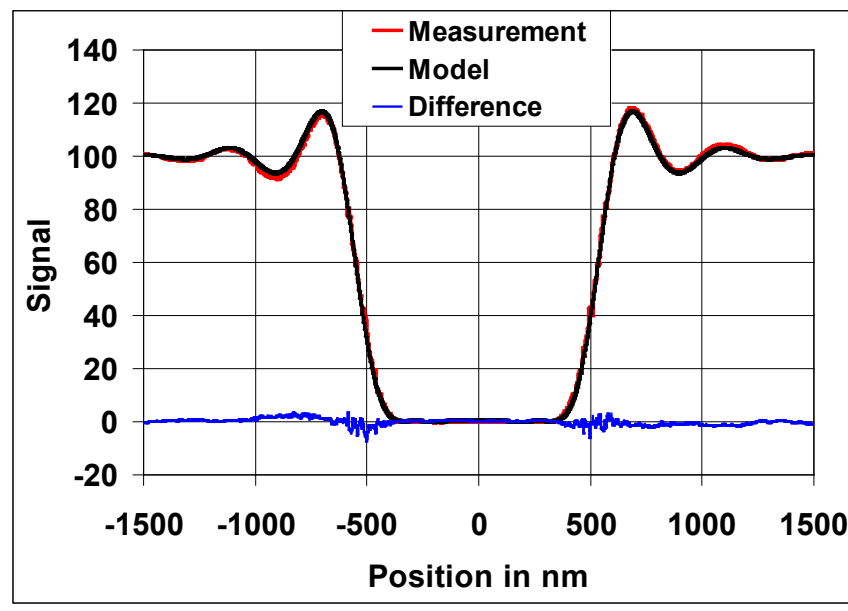

Fig. 9: a) Photo of the UV transmission microscope used at PTB for quantitative optical microscopy, primarily on photomasks, b) typical transmission intensity profile. 


\subsection{Scatterometry}

In scatterometry one analyses the whole optical diffraction spectrum of a measurement object in a non-imaging set-up and is thus not diffraction limited. The optical diffraction pattern of e.g. grating structures does not only contain information about the mean pitch, but also about other dimensional features of the grating structures like the height, top CD (line width) or bottom CD and side-wall angle, describing the geometrical profile of the surface structure. To extract information on these structure parameters from a measured optical diffraction pattern, the inverse diffraction problem has to be solved. For this task, the Maxwell solver program packages are applied which were already mentioned in the last section. Details about systematic effects in $\mathrm{CD}$ reconstruction from scatterometry data as well as the influence of line edge roughness were discussed in $[18,19]$. At PTB, two different scatterometry instruments were developed and are applied in the EUV (13 nm) [20] and the DUV (193 nm) [21] wavelength region, see fig. 10.

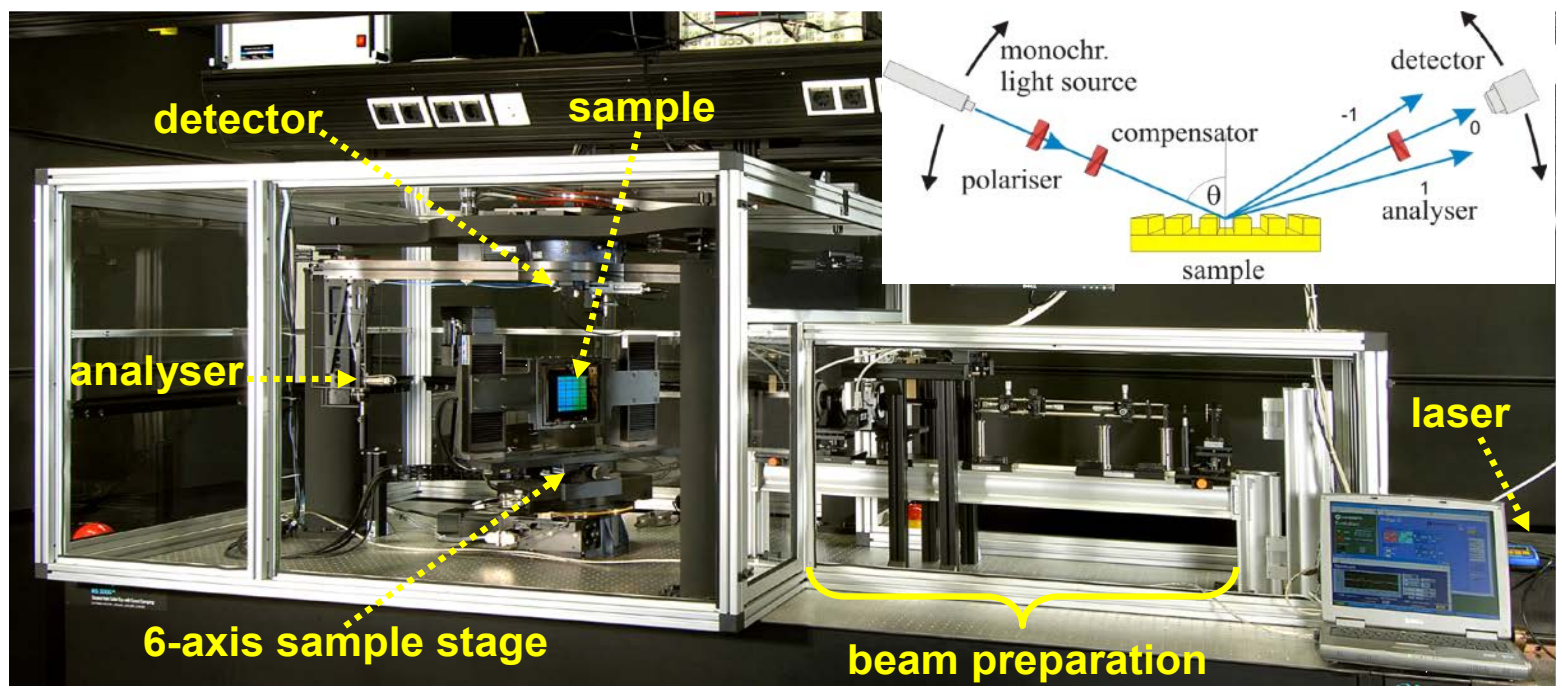

Fig. 10: Basic operation principle and photo of the new DUV scatterometer for mask metrology.

A systematic comparison between CD-AFM and EUV-scatterometry measurements on an EUV reflection mask was reported in [22]. Figure 11 shows the assumed model topography of the EUV reflection multilayer and the mask absorber stack, while fig. 12 shows the measurement results for $\mathrm{CD}$ and for sidewall angle of the absorber features.

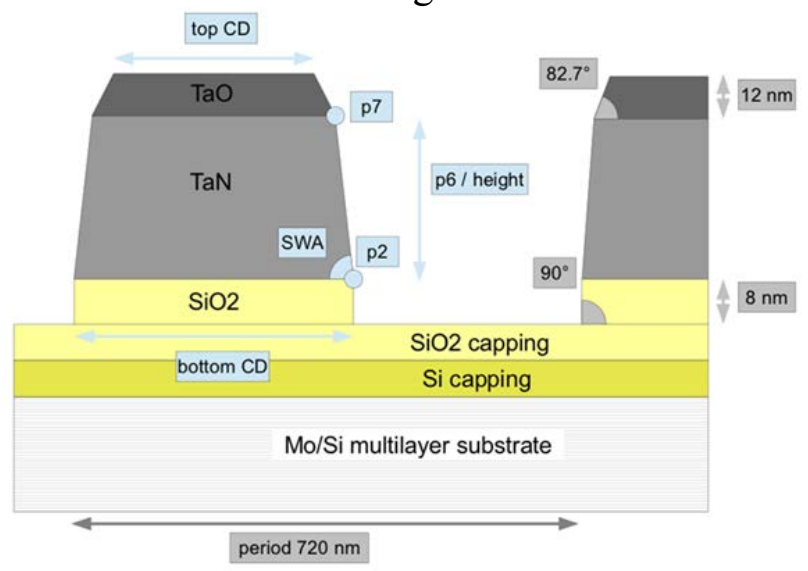

Fig. 11: Model topography used for solving the inverse problem in EUV scatterometry. 

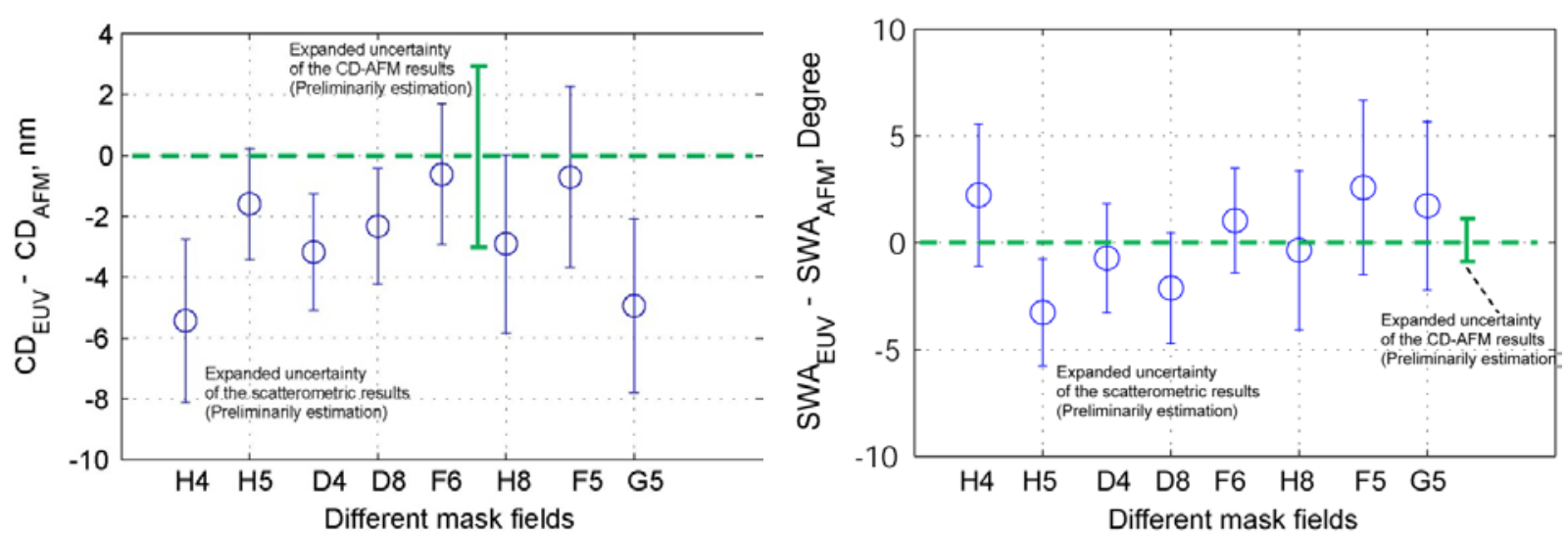

Fig. 12: Comparison of the measurement results obtained by the CD-AFM and EUV-scatterometer, shown as (a) the measured middle CD and (b) the measured sidewall angles. For clarity, the difference of the AFM and scatterometer results is plotted. The error bars given show the preliminarily estimated expanded uncertainty $(k=2)$ of both methods.

\subsection{Scanning Electron Microscopy}

Scanning electron microscopy (SEM) is widely used as a high resolution imaging tool in different areas of nanotechnology. Today, SEMs offer a size of the focused beam of about $1 \mathrm{~nm}$ even at comparatively low energies of about $1 \mathrm{keV}$. At these rather low energies, beam damages and electrostatic charging effects on the samples surfaces are largely reduced and the emission of secondary electrons, which are normally used for imaging is increased in this energy range of primary electrons [23]. For quantitative measurements of the size of nanostructures such as the width of lithographic lines, specially adapted so-called CD-SEM are used for quality control purposes in mask and wafer production. Special care is taken in operation of the CD-SEM to achieve optimum reproducibility of the sample positioning and the imaging process. Figure 13 shows the so-called Electron-Optical Metrology System (EOMS) of the PTB, which combines a low-voltage scanning electron microscopy of the Zeiss ULTRA type and a large vacuum chamber which contains a laser-interferometer controlled 2D precision stage for positioning of larger planar substrates [24]. The EOMS has been applied, for example, for high precision calibration of a grating sample with a nominal pitch of $100 \mathrm{~nm}$ [25].
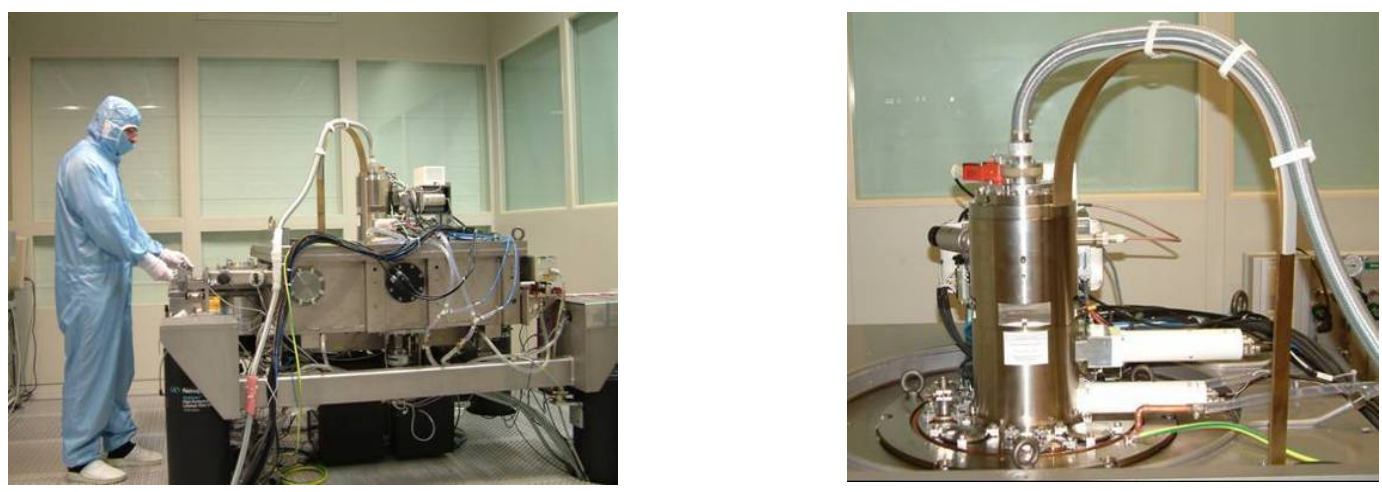

Fig. 13: Photo of the Electron Optical Metrology System (EOMS) in the PTB clean room facility (left) and of the ULTRA SEM mounted on top of the EOMS chamber (right).

If the sample stages of CD-SEM are not equipped with laser interferometric position feedback control, calibrated grating standards which are, in order to avoid Abbe errors, mounted on the same height as the samples to be measured can be used to determine the magnification of the 
raster scan area of the CD-SEM. If it is required to correlate the acquired signal contrast of an SEM image of a lithographic line feature with topographical details of the line feature, such as e.g. $C D$ at top of the feature or $C D$ at $50 \%$ height of the feature, one needs to apply a suitable physical model for the interaction of the primary electrons with the material and topography of the line feature to be investigated. Figure 14 shows the resulting simulated secondary electron profile at an edge of a smaller and a larger line feature. It is clear, that the data evaluation algorithms would have to take into account these different characteristics at the feature edges, if they are expected to extract traceable positions of topographic details of line features from measured SEM edge signals.

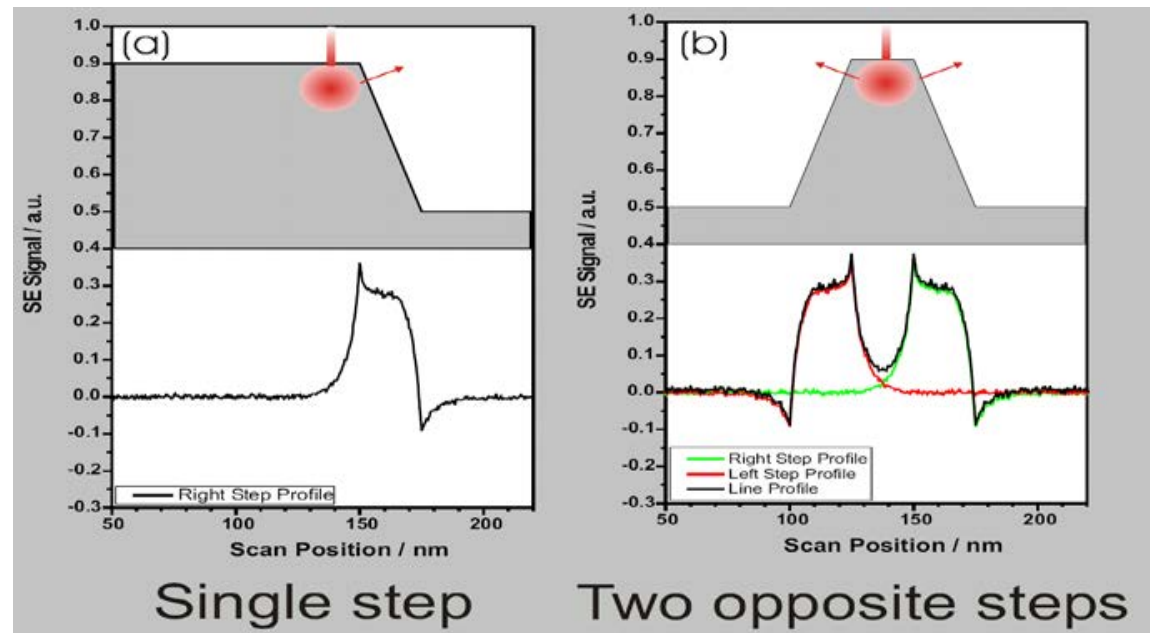

Fig. 14: Schematic cross sections of line features (large: a); small: b)) and simulated secondary electron profiles resulting from the interaction of the primary beam with the feature edge(s).

Different software program packages have been developed for this task. In order to take into account the stochastic nature of the different elastic and inelastic scattering events of the primary electrons as well as the resulting electrons (backscattered, secondary or transmitted electrons) in the solid state the majority of them uses Monte Carlo simulations. An overview of the applied models is provided in [26], and a detailed description of MCSEM, the modular Monte Carlo software package for simulation of electron signal contrast developed at the PTB, has been published in [27]. Uncertainties of CD-SEM measurements at PTB for the width of high quality lithographic features of sizes down to $100 \mathrm{~nm}$ and below on photomasks and wafers as low as $U_{95 \%}=10 \mathrm{~nm}$ have been reached. SEM in transmission mode has recently been successfully applied for traceable determination of the size and the size distribution of nanoparticle samples down to nanoparticle sizes of about $7 \mathrm{~nm}$ with uncertainties of about 1-2 $\mathrm{nm}$ [28].

\subsection{CD-AFM}

At the PTB different scanning probe microscopes are in use. Among them also is a large range metrological scanning probe microscope (LR-SPM) on the basis of a nanopositioning machine of company SIOS [29]. For traceable CD metrology on larger substrates such as photomasks or wafers, we are using a Nanostation 300 as the basic positioning machine, a scanning head based on a 6-axis flexure stage and a self-developed operation mode for probing the surface under test, the so-called vector approach probing [30]. Figure 15 shows the reproducibility of repeated measurements of the surface at the top and the sidewall of a lithographic Si line using a flared or reentrant CD tip, made with e-beam assisted deposition techniques (CDR-EBD tip) [31]. 

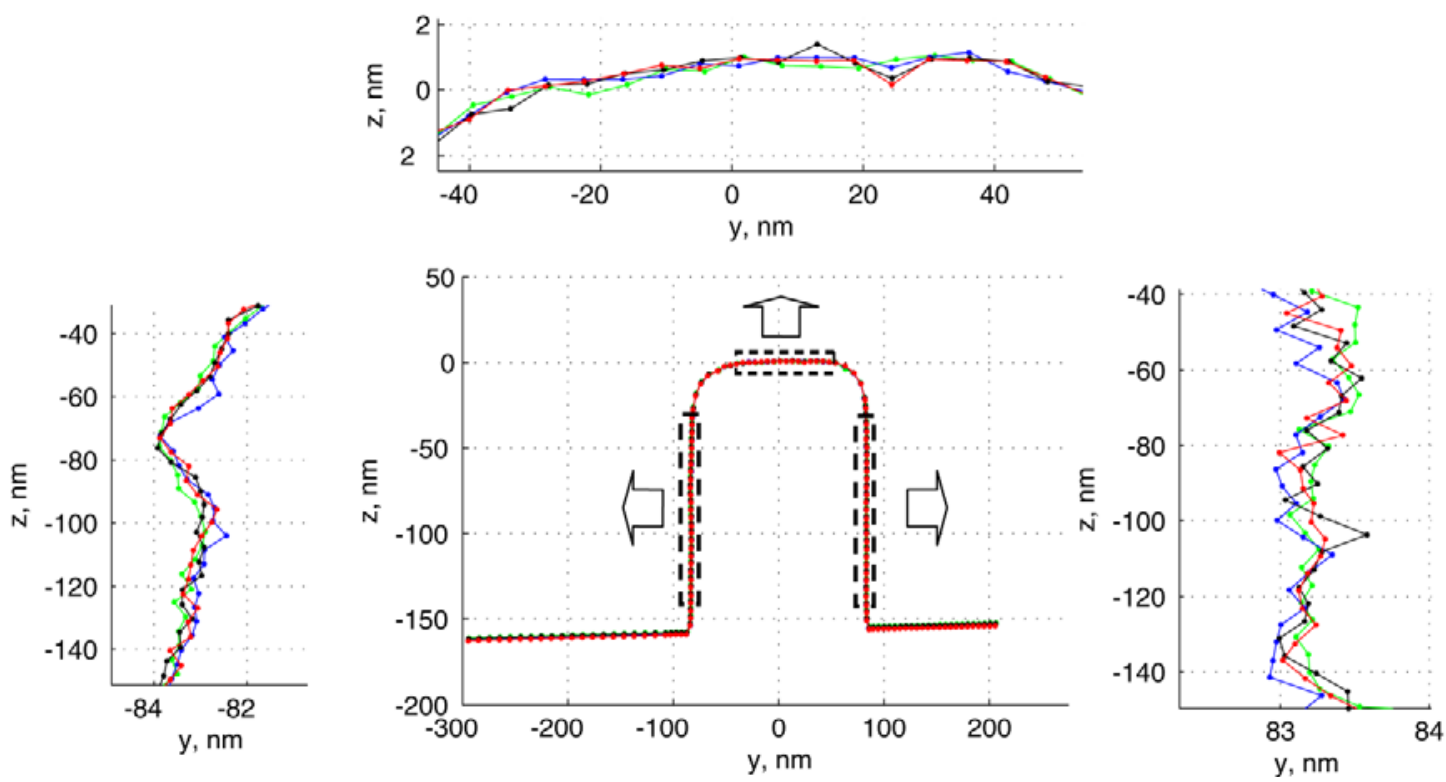

Fig. 15: Measurement repeatability of the CD-AFM using a CDR-EBD tip with a nominal tip width of $70 \mathrm{~nm}$.

\subsection{CD-AFM: Traceability via TEM measurements}

Based on the good reproducibility of the CD-AFM measurements on Si line features as demonstrated in fig. 15, we intended to obtain a traceable calibration of the width of the line feature itself. Here we followed an approach which has been realized before, namely to make use of the well known distances of surface atoms of single crystal silicon material [32]. We were using a different type of a commercially available silicon single crystal material standard with Si lines showing vertical sidewalls, the IVPS 100 standard available from [33]. The wafer sample contains several groups of vertical Si line features, which allows to perform CD-AFM comparison measurements between the features within the different groups. Afterwards a few features of the sample can be prepared to imaged in a high resolution transmission electron microscope (HR-TEM). Based on the known value of the Si lattice parameter, which has been determined by means of x-ray interferometry on Si bulk crystal material with very high accuracy before, the image magnification in a HR-TEM image can be calibrated and the width of a single crystal Si line feature can be inferred from counting the number of lattice planes and properly taking into account the silicon oxide layer at the edges of the Si line correctly. In addition to the Si line features whose lattice parameter can provide a calibration for the TEM image magnification, a second method was applied. It uses a double line feature being imaged in the HR-TEM image as well whose distance has been calibrated by a metrological SPM before, for details see [34]. Figure 16 shows the basic scheme of the metrological approach, which was taken here, while fig. 17 shows typical SEM and HR-TEM images of the prepared Si line features.

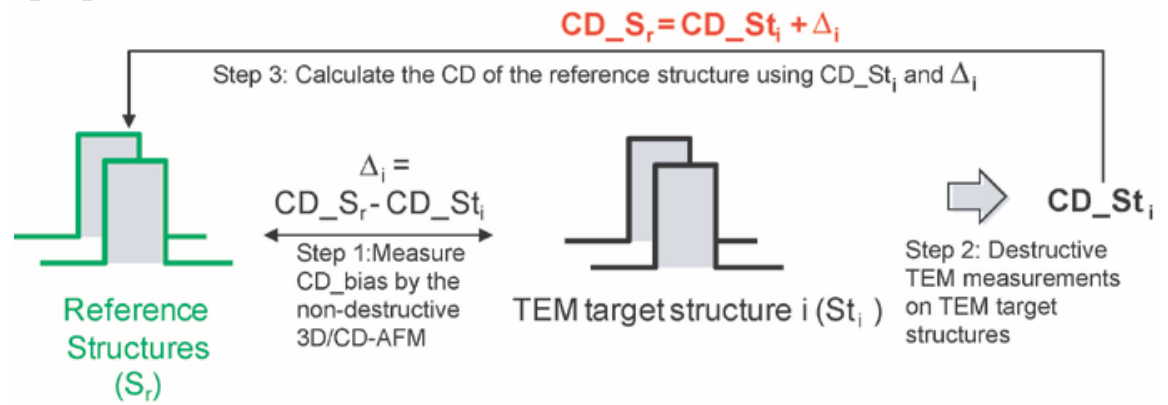

Fig. 16: Schematic diagram showing the strategy of disseminating the TEM results. 


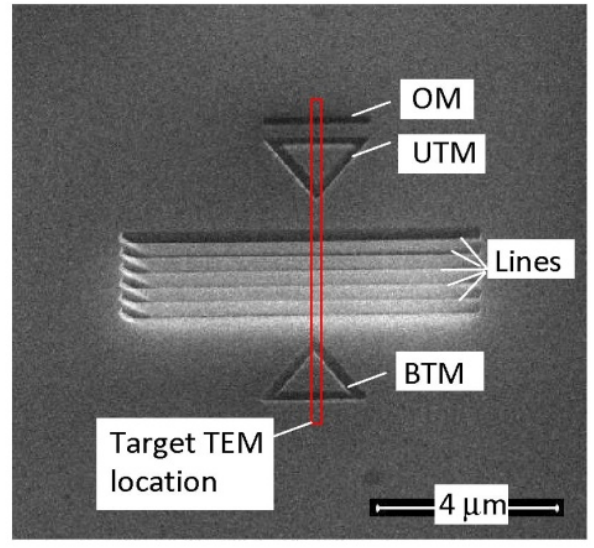

(a)

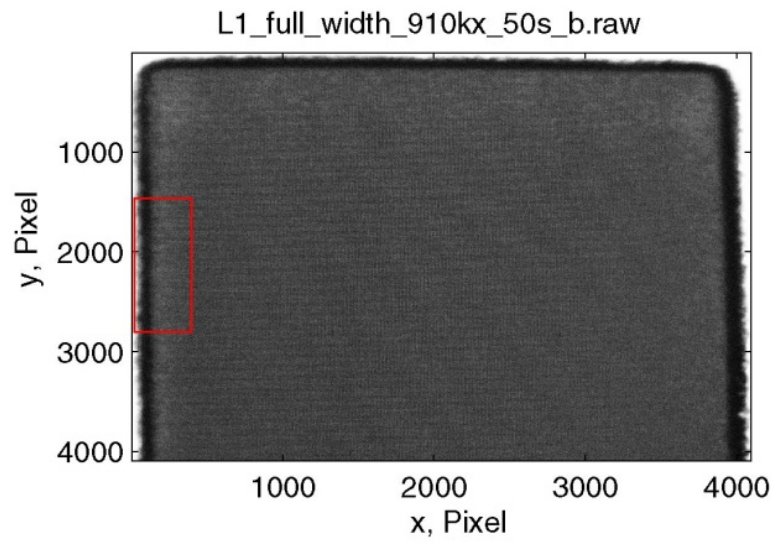

(c)

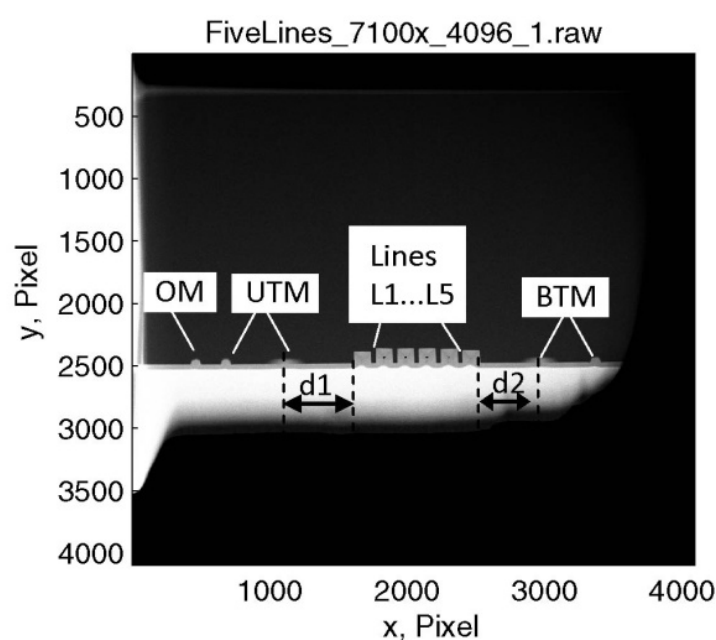

(b)

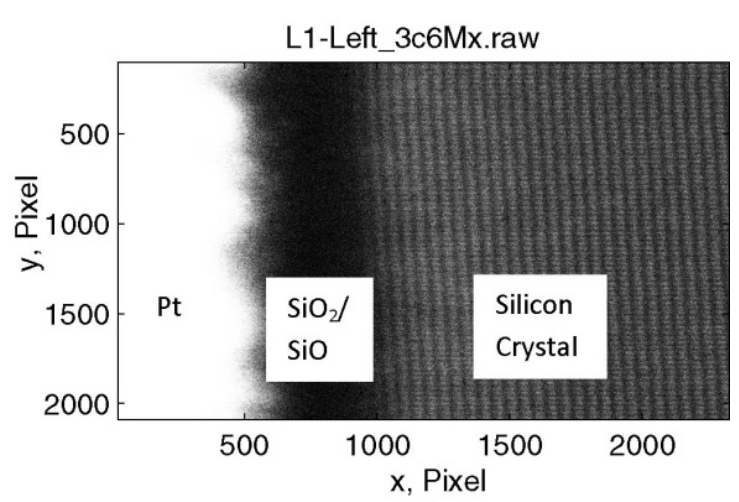

(d)

Fig. 17: (a) A SEM image showing the line feature pattern and the added alignment marks; (b) an overview STEM image obtained with a magnification of $7.1 \mathrm{k}$; (c) a typical HAADF TEM image measured on a CD reference structure (nominal CD of $100 \mathrm{~nm}$ ) with a magnification of $910 \mathrm{k}$; (d) a typical TEM image obtained with a higher magnification of $3.6 \mathrm{M}$ taken at the left part of the structure, as marked in (c).

The table 1 shows different measurement results for the calibration of the CD reference structure on the Si wafer, transferred from different TEM measurements. The CD results agree within $1.2 \mathrm{~nm}$, a first estimation of the measurement uncertainty for the CD value of the reference structure is $U_{95 \%}=1.6 \mathrm{~nm}$. One of the largest contributions in the uncertainty budget is the influence of the line edge roughness of the Si line standards, which shows the room for improvement in manufacturing quality of standards applied in nanotechnology.

\begin{tabular}{|c|c|c|c|c|}
\hline $\begin{array}{l}\text { Measure- } \\
\text { ment No. }\end{array}$ & $\begin{array}{l}\text { Registered } \\
\text { CD_Bias }\end{array}$ & $\begin{array}{l}\text { Applied } \\
\text { TEM } \\
\text { structure }\end{array}$ & $\begin{array}{l}\text { Measured } \\
\text { CD of TEM } \\
\text { structure }\end{array}$ & $\begin{array}{l}\mathrm{CD} \text { of the } \\
\text { reference } \\
\text { structure }\end{array}$ \\
\hline 1 & 11.0 & M11S1 & 96.7 & 107.7 \\
\hline 2 & 2.3 & M22S1 & 105.3 & 107.6 \\
\hline 3 & -0.9 & M78S1 & 108.1 & 107.2 \\
\hline 4 & -0.4 & M79S1 & 108.6 & 108.2 \\
\hline 5 & 4.3 & M71S1 & 102.7 & 107.0 \\
\hline
\end{tabular}

Table 1: Determined CD values of a PTB reference sample from five independent TEM target structures, all data in $\mathrm{nm}$. 


\section{SUMMARY AND OUTLOOK}

Recent developments of the PTB in high precision metrology of position and size of nanofeatures were described. It was shown, that the achievement of uncertainties in the $\mathrm{nm}$ range require both, special instrumentation and suitable physical models of the measurement signals. Further reductions of the measurement uncertainty for the position of nanofeatures on substrates larger than several $\mathrm{mm}$ would require improvements in the thermal drift behavior of instrument components and in the mounting of the substrates, while all measurements of position (small and large substrates) would benefit from improved manufacturing quality of the graduation line features. Further reductions of measurement uncertainty for the size of nanofeatures would require improvements in the manufacturing quality of standards as well as of the used signal modeling approaches.

\section{ACKNOWLEDGEMENTS}

The authors would like to acknowledge the support from PTB colleagues and external project partners, who contributed to the results shown in this paper.

\section{REFERENCES}

[1] R. Köning, J. Flügge, H. Bosse: Achievement of sub nanometer reproducibility in line scale measurements with the Nanometer Comparator, SPIE Advanced Lithography, 25 February - 2 March 2007, San Jose, California, USA

[2] Tiemann, I. et. al.: An international length comparison using vacuum comparators and a photoelectric incremental encoder as transfer standard, Prec. Eng. 32 (2008), p. 1-6.

[3] Köning, R., Weichert, Ch., Przebierala, B., Flügge, J., Häßler-Grohne, W., Bosse, H.: Implementing registration measurements on photomasks at the Nanometer Comparator, Measurement Science and Technology: 23 (2012), 9, 094010-1 - 094010-9

[4] Weichert, Ch., Stavridis, M., Walzel, M., Elster, C., Wiegmann, A., Schulz, M., Köning, R., Flügge, J., Tutsch, R.: A model based approach to reference-free straightness measurement at the Nanometer Comparator, Modeling aspects in optical metrology II ; (Proceedings of SPIE: 7390): (2009), 73900O-1 - 73900O-10

[5] Ch. Weichert: Upgrade des Nanometerkomparators zur Messung von Geradheit an Teilungen, PTB-Jahresbericht 2013

[6] Köchert, P., Flügge, J.; Köning, R., Weichert, Ch.; Manske, E.: Ultra-präzise Lageregelung mittels interferometrischer Positionsdetektion und Tauchspulenantrieb, Technisches Messen, 81 (2014), 6, 316-323

[7] On the possible future revision of the SI, http://www.bipm.org/en/si/new si/, accessed at July 28, 2014

[8] A. Birk et al.: Counting the atoms in ${ }^{28}$ Si crystal for a new kilogram definition, Metrologia: 48 (2011), S1 - S13

[9] Massa, E., Mana, G., Kuetgens, U., Ferroglio, L.: Measurement of the \{2 20$\}$ lattice-plane spacing of $a^{28}$ Si $x$-ray interferometer, Metrologia: 48 (2011), 2, S37 - S43

[10] Weichert, Ch., Köchert, P., Köning, R., Flügge, J., Andreas, B., Kuetgens, U., Yacoot, A.: A heterodyne interferometer with periodic nonlinearities smaller than $\pm 10 \mathrm{pm}$,

Measurement Science and Technology: 23 (2012), 9, 094005-1 - 094005-7

[11] E. Abbe: Beiträge zur Theorie des Mikroskops und der mikroskopischen Wahrnehmung, Schultzes Archiv f. mikr. Anat. 9, (1873), 413-468 
[12] M. Totzeck: Numerical simulation of high-NA quantitative polarisation microscopy and corresponding near-fields, Optik 112 (2001), 399-406

[13] http://www.wias-berlin.de/software/DIPOG/, (accessed on 22/07/2014)

[14] http://www.jcmwave.com/, (accessed on 22/07/2014)

[15] B. Bodermann, E. Buhr, G. Ehret, F. Scholze, M. Wurm: Optical metrology of microand nanostructures at PTB: Status and future developments, Proc. SPIE 7155, 71550V (2008)

[16] B. Bodermann, Z. Li, F. Pilarski, D. Bergmann: A 193nm microscope for CD metrology for the 32nm node and beyond, Proc. SPIE 7545 (2010), 75450A

[17] B. Bodermann, Bernd, E. Buhr, Z. Li, H. Bosse: Quantitative optical microscopy at the nanoscale: new developments and comparisons, in: Optical imaging and metrology: advanced technologies: (2012), 255 - 282, Wiley, ISBN 978-3-527-41064-4

[18] Kato, A., Burger, S., Scholze, F.: Analytical modeling and three-dimensional finite element simulation of line edge roughness in scatterometry, Applied Optics: 51 (2012), 27, $6457-6464$

[19] Henn, M.-A., Gross, H., Heidenreich, S., Scholze, F., Elster, C., Bär, M.: Improved reconstruction of critical dimensions in extreme ultraviolet scatterometry by modeling systematic errors, Measurement Science and Technology: 25 (2014), 4, 044003-1 - 044003-9 [20] Scholze, F. et al. (2008) Proc. Advanced Lithography Conf., SPIE 6921, 69213R-169213R-11.

[21] Wurm, M., Pilarski, F. and Bodermann, B. (2010): A new flexible scatterometer for critical dimension metrology, Rev. Sci. Instrum., Vol. 81, No. 2, pp.023701-1-023701-8, 023701.

[22] Dai, G., Hahm, K., Scholze, F., Henn, M.-A., Gross, H., Flügge, J., Bosse, H.: Measurements of CD and sidewall profile of EUV photomask structures using CD-AFM and tilting-AFM, Measurement Science and Technology: 25 (2014), 4, 044002-1 - 044002-13 [23] Reimer L.: Image Formation in Low-Voltage Scanning Electron Microscopy, SPIE, 1993 [24] W. Häßler-Grohne, H. Bosse: Electron optical metrology system for pattern placement measurements, Meas. Sci. Technol. 9, 1120-1128 (1998)

[25] W. Häßler-Grohne, T. Dziomba, C.G. Frase, H. Bosse, J. Prochazka: Characterization of a $100 \mathrm{~nm} 1 D$ pitch standard by metrological SEM and SFM, Proc. SPIE Microlithography, Vol. 5375, p. 426-436, 2004

[26] Frase, C. G., Gnieser, D., Bosse, H.: Model-based SEM for dimensional metrology tasks in semiconductor and mask industry, Journal of Physics D: 42 (2009), 18, 183001-1 - 18300117

[27] Johnsen, K.-P., Frase, C. G., Bosse, H. Gnieser, D.: SEM image modeling using the new Monte Carlo MCSEM, Metrology, inspection, and process control for microlithography XXIV; Proc. SPIE on CD-ROM: 7638, (2010), file name: 7638-59.pdf, 76381O-1 - 76381O-9 [28] Klein, T., Buhr, E., Frase, C. G.: TSEM - a review of scanning electron microscopy in transmission mode and its applications, in: Advances in imaging and electron physics: 171 (2012), 297 - 356, Elsevier, Amsterdam

[29] Dai, G., Wolff, H., Pohlenz, F., Danzebrink, H.-U.: A metrological large range atomic force microscope with improved performance, Review of Scientific Instruments: 80 (2009), 4, 043702-1 - 043702-10

[30] Dai, G., Häßler-Grohne, W., Hüser, D., Wolff, H., Danzebrink, H.-U., Koenders, L., Bosse, H.: Development of a 3D-AFM for true 3D measurements of nanostructures, Measurement Science and Technology: 22 (2011), 9, 094009-1 - 094009-10 [31] G. Dai, K. Hahm, F. Scholze, M.-A. Henn, H. Gross, J. Flügge, H. Bosse: Measurements of $C D$ and sidewall profile of EUV photomask structures using CD-AFM and Tilting-AFM, Measurement Science and Technology: 25 (2014), 4, 044002-1 - 044002-13 
[32] R. G. Dixson, R. A. Allen, W. F. Guthrie, and M. W. Cresswell: Traceable Calibration of Critical-Dimension Atomic Force Microscope Linewidth Measurements with Nanometer Uncertainty, J. Vac. Sci. Technol. B, Vol.23, 3028-3032 (2005)

[33] Team Nanotec GmbH, Wilhelm-Schickardt-Str. 10, 78052 Villingen-Schwenningen, Germany. Webpage: http://www.team-nanotec.de/index.cfm, accessed July 30, 2014 [34] Dai, G., Heidelmann, M., Kübel, Ch., Prang, R., Flügge, J., Bosse, H.: Reference nanodimensional metrology by scanning transmission electron microscopy, Measurement Science and Technology: 24 (2013), 8, 085001-1 - 085001-9

\section{CONTACTS}

Dr. rer. nat. Harald Bosse

Dr. rer nat. Bernd Bodermann

Dr. Gaoliang Dai

Dr.-Ing. Jens Flügge

Dr. rer. nat. Carl Georg Frase

Dipl.-Phys. Wolfgang Häßler-Grohne

Dipl.-Ing. Paul Köchert

Dr.-Ing. Rainer Köning

Dipl.-Phys. Christoph Weichert harald.bosse@ptb.de

bernd.bodermann@ptb.de

gaoliang.dai@ptb.de

jens.fluegge@ptb.de carl.g.frase@ptb.de

wolfgang.haessler-grohne@ptb.de

paul.koechert@ptb.de

rainer.koening@ptb.de

christoph.weichert@ptb.de 